\title{
Anomalous scattering factors of some rare earth elements evaluated using photon interaction cross-sections
}

\author{
S B APPAJI GOWDA, M L MALLIKARJUNA, R GOWDA and T K UMESH* \\ Department of Studies in Physics, Manasagangothri, University of Mysore, Mysore 570 006, India \\ ${ }^{*}$ Author for correspondence \\ Email: tku@uomphysics.net or tku1@yahoo.com
}

MS received 20 January 2003; accepted 1 April 2003

\begin{abstract}
The real and imaginary parts, $f^{\prime}(E)$ and $f^{\prime \prime}(E)$ of the dispersion corrections to the forward Rayleigh scattering amplitude (also called anomalous scattering factors) for the elements La, $\mathrm{Ce}, \mathrm{Pr}, \mathrm{Nd}, \mathrm{Sm}, \mathrm{Gd}$, Dy, Ho and Er, have been determined by a numerical evaluation of the dispersion integral that relates them through the optical theorem to the photoeffect cross-sections. The photoeffect cross-sections are derived from the total attenuation cross-section data set experimentally determined using high resolution high purity germanium detector in a narrow beam good geometry set-up for these elements in the photon energy range 5 to $1332 \mathrm{keV}$ and reported earlier by the authors. Below $5 \mathrm{keV}$, Scofield's photoeffect cross-sections compiled in XCOM program have been interpolated and used. Simple formulae for $f^{\prime \prime}$ in terms of atomic number and energy have also been obtained. The data cover the energy region from 6 to $85 \mathrm{keV}$ and atomic number $Z$ from 57-68. The results obtained are found to agree fairly well with the other available data.
\end{abstract}

Keywords. Rare earth elements; photoeffect; dispersion corrections $f^{\prime}$ and $f^{\prime \prime}$.

PACS Nos 32.80.t; 32.90.+a; 32.80.Fb; 32.80.Cy; 32.80.Hd

\section{Introduction}

Photoeffect is an important process of gamma-ray interaction below a few hundred keV. This process has been extensively studied theoretically as well as experimentally in view of its importance in some nuclear-physics, radiation-shielding, and astrophysical problems. Below $100 \mathrm{keV}$, the photoeffect is a major contributor to the attenuation of the photon beam incident on material targets.

The photoeffect cross-section $\tau$ is related to the dispersion correction to the forward angle Rayleigh scattering cross-section, through the optical theorem [1]. The scattering factor $f$ of an isolated atom relative to that of a free electron is given by

$$
f=f_{0}+f^{\prime}+i f^{\prime \prime}
$$

The first term $f_{0}$ is the atomic form factor and $f^{\prime}$ and $f^{\prime \prime}$ are the real and imaginary parts of dispersion corrections (also known as the anomalous scattering factors). $f^{\prime \prime}$ is 
related to the photoeffect cross-section through the relation based on the optical theorem [2] given by

$$
f^{\prime \prime}=\frac{E}{2 h c r_{0}} \sigma_{\mathrm{tot}},
$$

where $h c=12.398 \mathrm{keV} \AA$ in practical units, $r_{0}$ is the classical electron radius and $E$ is the energy of interest. Here, the total cross-section $\sigma_{\text {tot }}$ is given by $\sigma_{\text {tot }}=\tau+\sigma_{\mathrm{BBT}}-$ $\sigma_{\mathrm{BPP}}, \sigma_{\mathrm{BBT}}$ and $\sigma_{\mathrm{BPP}}$ are the photo excitation and bound pair production cross-sections respectively. For energies sufficiently away from absorption edges of a particular element, $\sigma_{\mathrm{BBT}}$ and $\sigma_{\mathrm{BPP}}$ are expected to be insignificant for $Z>10$, below the pair production threshold $[3,4]$.

In the energy region of current interest, if we neglect the spin flip processes, $f^{\prime}$ and $f^{\prime \prime}$ are connected by the modified Kramers-Kronig transform given by $[5,6]$

$$
f^{\prime}(E)=f^{\prime}(\infty)-\frac{2}{\pi} P \int_{0}^{\infty} \frac{E^{\prime} f^{\prime \prime}\left(E^{\prime}\right)}{E^{2}-E^{\prime 2}} \mathrm{~d} E^{\prime},
$$

where $f^{\prime}(\infty)$ is the high-energy limit, $P$ the Cauchy principal value of the dispersion integral and $E$ the energy of interest. Thus, it is possible to determine the dispersion corrections $f^{\prime}$ and $f^{\prime \prime}$ from a set of photoeffect cross-sections using (2) and (3).

A survey of literature on the evaluation of the dispersion corrections indicates that, right since the first theoretical prediction of X-ray dispersion by Waller [7], there have been a number of attempts to calculate the dispersion corrections $f^{\prime}$ and $f^{\prime \prime}$ [8-10].

Calculations of $f^{\prime}$ and $f^{\prime \prime}$ based on Waller's theory were made by Hönl [11] for hydrogen-like atoms in a non-relativistic approach. A comprehensive account of the work done thus far is found in [12-14].

Cromer and Liberman [15] have given extensive tabulations of dispersion corrections over a range of wavelengths commonly used by crystallographers. They used the selfconsistent field relativistic Dirac-Slater wave functions along with the inclusion of a relativistic correction term $5 / 3\left(E_{\text {tot }} / m c^{2}\right)$ (high energy limit $f^{\prime}(\infty)$ ) in the dipole approximation.

Creagh [16] and Smith [17] pointed out that when higher order multipolar expansions as well as retardation effects are considered, the relativistic correction becomes $E_{\text {tot }} / m c^{2}$. Creagh and McAuley [18] have given tabulations of $f^{\prime}$ and $f^{\prime \prime}$ which make use of this correction term.

Kissel et al [19] have developed a computer program based on the second order $S$-matrix formalism to predict the total atom Rayleigh scattering amplitudes. Kissel and Pratt [20] have tabulated the values of the high-energy limit, based on the $S$-matrix calculations, to be added to $f^{\prime}$ and also given the values of correction to be added to the Cromer-Liberman $f^{\prime}$ values. The values of dispersion corrections predicted by them are commensurate with those of Creagh and McAuley [18] for very low $Z$ elements and are different by about $6 \%$ to $7 \%$ for $Z>20$.

Chantler [21] has provided extensive tabulations of theoretical form factor, attenuation and scattering data for elements of $Z=1$ to 92 from $E=1-10 \mathrm{eV}$ to $E=0.4-1 \mathrm{MeV}$. Here, the primary interactions of $\mathrm{X}$-rays with isolated atoms are described and computed within the self-consistent Dirac-Hartee-Fock framework. The relativistic correction term used was the one calculated by Creagh and McAuley [18]. 
Recently, Cullen et al [22] have provided a photon data library (EPDL97) which is available from the IAEA on a CD-ROM. This library includes photon interaction data for all elements with atomic number between 1 and 100 over the range $1 \mathrm{eV}$ to $100 \mathrm{GeV}$. $f^{\prime}$ and $f^{\prime \prime}$ values have also been provided. The relativistic correction term used was the one calculated by Kissel and Pratt [20].

Henke et al [6] have given tabulations of the dispersion corrections calculated for all $\mathrm{Z}$ in the energy range $30 \mathrm{eV}$ to $30 \mathrm{keV}$, based on a semi-empirical approach using theoreticalexperimental attenuation coefficient data base.

On the experimental side, it can be noticed that two types of techniques have been mainly employed to determine the values of $f^{\prime}$ and $f^{\prime \prime}$. These are: (1) direct method and (2) attenuation coefficient method.

The direct method has been widely used by several investigators to evaluate $f^{\prime}$ and $f^{\prime \prime}$. This method is based on (i) the measurement of the refractive index of the specimen of interest, (ii) the determination of intensities of Bragg reflections [23] and (iii) X-ray interferometry [24] from which $f^{\prime}$ and $f^{\prime \prime}$ values are calculated. The principal advantage of this method is that it can yield absolute values of $f^{\prime}$ and $f^{\prime \prime}$. The main drawback is that the range of X-ray wavelengths and atomic numbers that can be covered by this method is limited. Also, there are problems associated with specimen dimensions and device stability.

The dispersion corrections $f^{\prime}$ and $f^{\prime \prime}$ can also be evaluated by the attenuation coefficient method. Attenuation experiments do not suffer from the problems associated with specimen dimensions and device stability. Measurements can be made for a larger range of atomic numbers over a wide range of energies [25]. This method has been employed by several investigators [26-29] to calculate $f^{\prime}$ and $f^{\prime \prime}$.

In our laboratory, Sandiago et al [30] have evaluated the dispersion corrections for the elements $\mathrm{Cu}$ and $\mathrm{Ag}$. Umesh et al [31] have evaluated the dispersion corrections for the elements $\mathrm{Zr}$ and $\mathrm{Sn}$ in the energy range 5-85 keV using the attenuation coefficient method.

In this paper, we report the dispersion corrections for some rare earth elements like La, Ce, Pr, Nd, Sm, Gd, Dy, Ho and Er, calculated using $f^{\prime \prime}$ values. The $f^{\prime \prime}$ values were evaluated from the photoeffect cross-sections using the attenuation coefficient method. The photoeffect cross-sections were derived from the measured total attenuation cross-section of the element by subtracting a small contribution of the sum of coherent and incoherent scattering cross-section interpolated from the XCOM data [32] for the energy of present interest. The total attenuation cross-sections were measured in the energy range 6 to 85 $\mathrm{keV}$ in a narrow beam good geometry set-up, by employing a high-resolution hyper-pure germanium detector [33]. Beyond $85 \mathrm{keV}$, experimental data measured earlier by two of the authors [34-37] were used. Below $6 \mathrm{keV}$, Scofield's [38] data were used. Simple formulae in terms of $Z$ and $E$ have also been obtained, by which $f^{\prime \prime}$ values can be obtained, below, above or at the $K$-edge of any element in the atomic number range 57 to 68 for all energies in the range $10-100 \mathrm{keV}$. The values of $f^{\prime}(E)$ and $f^{\prime \prime}(E)$ calculated using our experimental values for rare earth elements are compared with other available data and discussed.

\section{Experimental details}

The details regarding the experimental set-up used, the method of data acquisition, analysis and error discussion along with the calculation of the total attenuation cross-section of these elements have been reported earlier by the authors [33-37]. 


\section{Results and discussion}

\subsection{Calculation of imaginary part of the anomalous scattering factor $f^{\prime \prime}(E)$}

The photoeffect cross-sections $\tau$ of the rare earth elements were derived from the measured total attenuation cross-section of the element by subtracting small contribution of the sum of coherent and incoherent scattering cross-section interpolated from the XCOM data for the energy of present interest. The values of the photoeffect cross-section were used to calculate $f^{\prime \prime}(E)$ from eq. (2) at each energy of interest. The variation of $f^{\prime \prime}$ values as a function of atomic number $Z$ and energy $E$ was studied. From this, simple formulae for $f^{\prime \prime}$ in terms of $Z$ and $E$ were obtained. These formulae are shown in (4).

$$
\begin{aligned}
& 10^{4} f^{\prime \prime}=1.27742 E^{-2.303} Z^{(3.66003+0.14067 \ln E)} \quad \text { (below } K \text {-edge), } \\
& 10^{2} f^{\prime \prime}=3.217614 E^{-2.52178} Z^{(2.70699+0.20188 \ln E)} \quad \text { (above } K \text {-edge), } \\
& f^{\prime \prime}(E k)=5.42767 E_{k}^{-0.16793} \quad(\text { at } K \text {-edge }) \text {. }
\end{aligned}
$$

It was interesting to note that these formulae (eqs $(4 a)-(4 c)$ ), can reproduce $f^{\prime \prime}$ values not only below and above the $K$-edge but also at the $K$-edge energy of each element. The values of $\tau$ and $f^{\prime \prime}$ are listed in tables 1 and 2 along with the other available data for comparison. A good agreement can be observed among the respective values.

\subsection{Calculation of real part of the anomalous scattering factor $f^{\prime}(E)$}

The $f^{\prime \prime}$ values were used to calculate $f^{\prime}(E)$ by the numerical evaluation of the dispersion integral in (3). For this purpose, the lower limit of integration was chosen to be the $M_{V}$ absorption edge and the upper limit was $1332 \mathrm{keV}$. Below $6 \mathrm{keV}$ up to the $M_{V}$ edge, photoeffect cross-section data based on XCOM were used. Above $6 \mathrm{keV}$, those values of $\tau$ which were reported earlier by the authors were used. To evaluate the integral numerically, the energy region used for integration was divided into a large number of small intervals. Within each interval $\left(E_{i}, E_{i+1}\right)$, the energy dependence of $f_{i}^{\prime \prime}(E)$ was determined by a linear function

$$
f_{i}^{\prime \prime}(E)=a_{i}+b_{i} E
$$

In this interval, the dispersion integral assumes the form

$$
I_{i, i+1}\left(E_{S}\right)=\frac{2}{\pi} P \int_{E_{i}}^{E_{i+1}} \frac{E^{\prime} f^{\prime \prime}\left(E^{\prime}\right)}{E_{s}^{2}-E^{\prime 2}} \mathrm{~d} E^{\prime} .
$$

Mathematically, expression (6) is equivalent to

$$
\begin{aligned}
I_{i, i+1}\left(E_{S}\right)= & -\frac{2}{\pi}\left[\frac{a_{i}}{2} \ln \left|\frac{E_{s}^{2}-E_{i+1}^{2}}{E_{s}^{2}-E_{i}^{2}}\right|\right. \\
& \left.+b_{i}\left(E_{i+1}-E_{i}-\frac{E_{s}}{2} \ln \left|\frac{\left(E_{i+1}+E_{S}\right)\left(E_{i}-E_{s}\right)}{\left(E_{i+1}-E_{S}\right)\left(E_{i}+E_{S}\right)}\right|\right)\right] .
\end{aligned}
$$


Anomalous scattering factors of some rare earth elements

Table 1. Photoeffect cross-sections in elements (experimental errors are to the extent of 3-4\%; all cross-sections are in $10^{3}$ barn /atom).

\begin{tabular}{|c|c|c|c|c|c|c|c|c|c|}
\hline $\begin{array}{l}\text { Energy } \\
(\mathrm{keV})\end{array}$ & $\mathrm{La}$ & $\mathrm{Ce}$ & $\operatorname{Pr}$ & $\mathrm{Nd}$ & $\mathrm{Sm}$ & $\mathrm{Gd}$ & Dy & Ho & $\mathrm{Er}$ \\
\hline 6.4 & $\begin{array}{l}144.53 \\
141.50 \\
140.14\end{array}$ & - & - & - & - & - & - & - & - \\
\hline 8.041 & $\begin{array}{l}78.52 \\
79.46 \\
78.48\end{array}$ & $\begin{array}{l}85.25 \\
84.80 \\
83.91\end{array}$ & $\begin{array}{l}90.90 \\
90.27 \\
91.80\end{array}$ & $\begin{array}{l}96.82 \\
95.81 \\
94.85\end{array}$ & - & - & - & - & - \\
\hline 10.53 & $\begin{array}{l}38.14 \\
38.90 \\
38.41\end{array}$ & $\begin{array}{l}41.18 \\
41.55 \\
41.07\end{array}$ & $\begin{array}{l}43.98 \\
44.37 \\
43.79\end{array}$ & $\begin{array}{l}46.91 \\
47.32 \\
46.63\end{array}$ & $\begin{array}{l}53.21 \\
53.66 \\
52.78\end{array}$ & $\begin{array}{l}60.13 \\
60.53 \\
59.49\end{array}$ & $\begin{array}{l}67.63 \\
67.52 \\
67.20\end{array}$ & $\begin{array}{l}71.65 \\
71.19 \\
70.42\end{array}$ & $\begin{array}{l}75.83 \\
75.05 \\
75.86\end{array}$ \\
\hline 14.4 & $\begin{array}{l}16.39 \\
16.80 \\
16.23\end{array}$ & $\begin{array}{l}17.63 \\
17.99 \\
17.45\end{array}$ & $\begin{array}{l}18.86 \\
19.26 \\
18.72\end{array}$ & $\begin{array}{l}20.15 \\
20.57 \\
20.06\end{array}$ & $\begin{array}{l}22.94 \\
23.38 \\
22.86\end{array}$ & $\begin{array}{l}26.02 \\
26.47 \\
25.85\end{array}$ & $\begin{array}{l}29.35 \\
29.78 \\
29.06\end{array}$ & $\begin{array}{l}31.15 \\
31.53 \\
30.83\end{array}$ & $\begin{array}{l}33.02 \\
33.35 \\
33.03\end{array}$ \\
\hline 24.14 & $\begin{array}{l}4.00 \\
4.06 \\
3.99\end{array}$ & $\begin{array}{l}4.27 \\
4.36 \\
4.29\end{array}$ & $\begin{array}{l}4.58 \\
4.68 \\
4.59\end{array}$ & $\begin{array}{l}4.92 \\
5.01 \\
4.92\end{array}$ & $\begin{array}{l}5.64 \\
5.74 \\
5.64\end{array}$ & $\begin{array}{l}6.44 \\
6.53 \\
6.43\end{array}$ & $\begin{array}{l}7.31 \\
7.40 \\
7.29\end{array}$ & $\begin{array}{l}7.78 \\
7.87 \\
7.76\end{array}$ & $\begin{array}{l}8.27 \\
8.35 \\
8.27\end{array}$ \\
\hline 30.8 & $\begin{array}{l}2.04 \\
2.06 \\
2.03\end{array}$ & $\begin{array}{l}2.16 \\
2.22 \\
2.16\end{array}$ & $\begin{array}{l}2.33 \\
2.38 \\
2.18\end{array}$ & $\begin{array}{l}2.50 \\
2.55 \\
2.51\end{array}$ & $\begin{array}{l}2.88 \\
2.93 \\
2.89\end{array}$ & $\begin{array}{l}3.30 \\
3.35 \\
3.30\end{array}$ & $\begin{array}{l}3.76 \\
3.80 \\
3.92\end{array}$ & $\begin{array}{l}4.01 \\
4.04 \\
3.99\end{array}$ & $\begin{array}{l}4.27 \\
4.30 \\
4.27\end{array}$ \\
\hline 35 & $\begin{array}{l}1.42 \\
1.44 \\
1.38\end{array}$ & $\begin{array}{l}1.51 \\
1.55 \\
1.49\end{array}$ & $\begin{array}{l}1.62 \\
1.66 \\
1.61\end{array}$ & $\begin{array}{l}1.75 \\
1.79 \\
1.73\end{array}$ & $\begin{array}{l}2.02 \\
2.05 \\
2.01\end{array}$ & $\begin{array}{l}2.32 \\
2.34 \\
2.31\end{array}$ & $\begin{array}{l}2.65 \\
2.67 \\
2.63\end{array}$ & $\begin{array}{l}2.82 \\
2.84 \\
2.80\end{array}$ & $\begin{array}{l}3.01 \\
3.02 \\
3.01\end{array}$ \\
\hline 52.01 & $\begin{array}{l}2.88 \\
2.91 \\
2.88\end{array}$ & $\begin{array}{l}3.08 \\
3.08 \\
3.06\end{array}$ & $\begin{array}{l}3.27 \\
3.27 \\
3.25\end{array}$ & $\begin{array}{l}3.46 \\
3.45 \\
3.45\end{array}$ & $\begin{array}{l}3.88 \\
3.88 \\
3.86\end{array}$ & $\begin{array}{l}4.33 \\
4.31 \\
4.28\end{array}$ & $\begin{array}{l}0.87 \\
0.88 \\
0.86\end{array}$ & $\begin{array}{l}0.93 \\
0.94 \\
0.92\end{array}$ & $\begin{array}{l}1.00 \\
1.01 \\
1.00\end{array}$ \\
\hline 64.3 & $\begin{array}{l}1.64 \\
1.64 \\
1.52\end{array}$ & $\begin{array}{l}1.74 \\
1.75 \\
1.23\end{array}$ & $\begin{array}{l}1.85 \\
1.86 \\
1.84\end{array}$ & $\begin{array}{l}1.96 \\
1.97 \\
1.95\end{array}$ & $\begin{array}{l}2.20 \\
2.21 \\
2.20\end{array}$ & $\begin{array}{l}2.46 \\
2.46 \\
2.46\end{array}$ & $\begin{array}{l}2.75 \\
2.73 \\
2.73\end{array}$ & $\begin{array}{l}2.90 \\
2.88 \\
2.88\end{array}$ & $\begin{array}{l}3.05 \\
3.06 \\
3.05\end{array}$ \\
\hline 70.833 & $\begin{array}{l}1.26 \\
1.26 \\
1.25\end{array}$ & $\begin{array}{l}1.34 \\
1.34 \\
1.33\end{array}$ & $\begin{array}{l}1.42 \\
1.43 \\
1.41\end{array}$ & $\begin{array}{l}1.51 \\
1.52 \\
1.50\end{array}$ & $\begin{array}{l}1.70 \\
1.70 \\
1.69\end{array}$ & $\begin{array}{l}1.90 \\
1.90 \\
1.90\end{array}$ & $\begin{array}{l}2.12 \\
2.12 \\
2.11\end{array}$ & $\begin{array}{l}2.24 \\
2.24 \\
2.22\end{array}$ & $\begin{array}{l}2.36 \\
2.37 \\
2.36\end{array}$ \\
\hline 72.87 & $\begin{array}{l}1.17 \\
1.16 \\
1.16\end{array}$ & $\begin{array}{l}1.24 \\
1.24 \\
1.23\end{array}$ & $\begin{array}{l}1.32 \\
1.32 \\
1.31\end{array}$ & $\begin{array}{l}1.40 \\
1.41 \\
1.39\end{array}$ & $\begin{array}{l}1.57 \\
1.58 \\
1.57\end{array}$ & $\begin{array}{l}1.76 \\
1.76 \\
1.76\end{array}$ & $\begin{array}{l}1.97 \\
1.97 \\
1.96\end{array}$ & $\begin{array}{l}2.07 \\
2.08 \\
2.07\end{array}$ & $\begin{array}{l}2.19 \\
2.20 \\
2.19\end{array}$ \\
\hline 81 & $\begin{array}{l}0.88 \\
0.88 \\
0.86\end{array}$ & $\begin{array}{l}0.93 \\
0.93 \\
0.92\end{array}$ & $\begin{array}{l}0.99 \\
0.99 \\
0.98\end{array}$ & $\begin{array}{l}1.05 \\
1.05 \\
1.05\end{array}$ & $\begin{array}{l}1.18 \\
1.18 \\
1.18\end{array}$ & $\begin{array}{l}1.32 \\
1.33 \\
1.32\end{array}$ & $\begin{array}{l}1.48 \\
1.49 \\
1.48\end{array}$ & $\begin{array}{l}1.56 \\
1.57 \\
1.56\end{array}$ & $\begin{array}{l}1.65 \\
1.65 \\
1.65\end{array}$ \\
\hline 84.3 & $\begin{array}{l}0.79 \\
0.78 \\
0.77\end{array}$ & $\begin{array}{l}0.83 \\
0.83 \\
0.83\end{array}$ & $\begin{array}{l}0.88 \\
0.88 \\
0.88\end{array}$ & $\begin{array}{l}0.94 \\
0.94 \\
0.94\end{array}$ & $\begin{array}{l}1.06 \\
1.06 \\
1.06\end{array}$ & $\begin{array}{l}1.19 \\
1.19 \\
1.19\end{array}$ & $\begin{array}{l}1.33 \\
1.33 \\
1.33\end{array}$ & $\begin{array}{l}1.40 \\
1.41 \\
1.40\end{array}$ & $\begin{array}{l}1.48 \\
1.48 \\
1.48\end{array}$ \\
\hline
\end{tabular}

First line: Present values, second line: XCOM values [32], third line: Chantler values [21]. 
S B Appaji Gowda et al

Table 2. Imaginary part of the anomalous scattering factor $\left(f^{\prime \prime}\right)$.

\begin{tabular}{|c|c|c|c|c|c|c|c|c|c|}
\hline $\begin{array}{l}\text { Energy } \\
(\mathrm{keV})\end{array}$ & $\mathrm{La}$ & $\mathrm{Ce}$ & $\operatorname{Pr}$ & $\mathrm{Nd}$ & $\mathrm{Sm}$ & $\mathrm{Gd}$ & Dy & Ho & $\mathrm{Er}$ \\
\hline 6.4 & $\begin{array}{l}13.638 \\
12.836 \\
12.997\end{array}$ & - & - & - & - & - & - & - & - \\
\hline 8.041 & $\begin{array}{l}9.1800 \\
9.0314 \\
9.1160\end{array}$ & $\begin{array}{l}9.8333 \\
9.6564 \\
9.7154\end{array}$ & $\begin{array}{l}10.521 \\
10.564 \\
10.344\end{array}$ & $\begin{array}{l}11.244 \\
10.915 \\
11.002\end{array}$ & - & - & - & - & \\
\hline 10.53 & $\begin{array}{l}5.7508 \\
5.7889 \\
5.8126\end{array}$ & $\begin{array}{l}6.1641 \\
6.1891 \\
6.6480\end{array}$ & $\begin{array}{l}6.5994 \\
6.5986 \\
6.6936\end{array}$ & $\begin{array}{l}7.0573 \\
7.0267 \\
7.1387\end{array}$ & $\begin{array}{l}8.0440 \\
7.9539 \\
8.0763\end{array}$ & $\begin{array}{l}9.1307 \\
8.9645 \\
9.1071\end{array}$ & $\begin{array}{l}10.324 \\
10.127 \\
10.191\end{array}$ & $\begin{array}{l}10.962 \\
10.612 \\
10.756\end{array}$ & $\begin{array}{l}11.630 \\
11.233 \\
11.354\end{array}$ \\
\hline 14.4 & $\begin{array}{l}3.3418 \\
3.3453 \\
3.4682\end{array}$ & $\begin{array}{l}3.5848 \\
3.5961 \\
3.7139\end{array}$ & $\begin{array}{l}3.8408 \\
3.8583 \\
3.9667\end{array}$ & $\begin{array}{l}4.1103 \\
4.1336 \\
4.2428\end{array}$ & $\begin{array}{l}4.6918 \\
4.7119 \\
4.8237\end{array}$ & $\begin{array}{l}5.3330 \\
5.3276 \\
5.4599\end{array}$ & $\begin{array}{l}6.0381 \\
5.9885 \\
6.1445\end{array}$ & $\begin{array}{l}6.4158 \\
6.3542 \\
6.5049\end{array}$ & $\begin{array}{l}6.8111 \\
6.7231 \\
6.8817\end{array}$ \\
\hline 24.14 & $\begin{array}{l}1.3641 \\
1.3791 \\
1.4122\end{array}$ & $\begin{array}{l}1.4652 \\
1.4809 \\
1.5089\end{array}$ & $\begin{array}{l}1.5717 \\
1.5874 \\
1.6185\end{array}$ & $\begin{array}{l}1.6841 \\
1.7003 \\
1.7432\end{array}$ & $\begin{array}{l}1.9269 \\
1.9483 \\
2.0025\end{array}$ & $\begin{array}{l}2.1954 \\
2.2201 \\
2.2837\end{array}$ & $\begin{array}{l}0.4912 \\
2.5196 \\
2.5619\end{array}$ & $\begin{array}{l}0.6499 \\
2.6795 \\
2.7426\end{array}$ & $\begin{array}{l}2.8162 \\
2.8468 \\
2.8995\end{array}$ \\
\hline 30.8 & $\begin{array}{l}0.8940 \\
0.8831 \\
0.9131\end{array}$ & $\begin{array}{l}0.9608 \\
0.9538 \\
0.9795\end{array}$ & $\begin{array}{l}1.0313 \\
0.9625 \\
1.0512\end{array}$ & $\begin{array}{l}1.1057 \\
1.1061 \\
1.1331\end{array}$ & $\begin{array}{l}1.2665 \\
1.2742 \\
1.2989\end{array}$ & $\begin{array}{l}1.4445 \\
1.4551 \\
1.4883\end{array}$ & $\begin{array}{l}1.6409 \\
1.7255 \\
1.6175\end{array}$ & $\begin{array}{l}1.7463 \\
1.7574 \\
1.7995\end{array}$ & $\begin{array}{l}1.8569 \\
1.8675 \\
1.8991\end{array}$ \\
\hline 35 & $\begin{array}{l}0.7163 \\
0.6921 \\
0.7210\end{array}$ & $\begin{array}{l}0.7700 \\
0.7474 \\
0.7762\end{array}$ & $\begin{array}{l}0.8267 \\
0.8064 \\
0.8341\end{array}$ & $\begin{array}{l}0.8866 \\
0.8689 \\
0.8983\end{array}$ & $\begin{array}{l}1.0162 \\
1.0064 \\
1.0338\end{array}$ & $\begin{array}{l}1.1597 \\
1.1555 \\
1.1858\end{array}$ & $\begin{array}{l}1.3181 \\
1.3164 \\
1.3406\end{array}$ & $\begin{array}{l}0.4032 \\
1.4009 \\
1.4362\end{array}$ & $\begin{array}{l}1.4924 \\
1.4895 \\
1.5176\end{array}$ \\
\hline 52.01 & $\begin{array}{l}2.1566 \\
2.1446 \\
2.1636\end{array}$ & $\begin{array}{l}2.2921 \\
2.2802 \\
2.2943\end{array}$ & $\begin{array}{l}2.4336 \\
2.4196 \\
2.4334\end{array}$ & $\begin{array}{l}2.5813 \\
2.5653 \\
2.5762\end{array}$ & $\begin{array}{l}2.8957 \\
2.8714 \\
2.8851\end{array}$ & $\begin{array}{l}3.2365 \\
3.1883 \\
3.1996\end{array}$ & $\begin{array}{l}0.6686 \\
0.6362 \\
0.6576\end{array}$ & $\begin{array}{l}0.7124 \\
0.6812 \\
0.7016\end{array}$ & $\begin{array}{l}0.7588 \\
0.7279 \\
0.7485\end{array}$ \\
\hline 64.3 & $\begin{array}{l}1.5019 \\
1.3947 \\
1.5067\end{array}$ & $\begin{array}{l}1.5975 \\
1.5910 \\
1.6054\end{array}$ & $\begin{array}{l}0.6974 \\
1.6928 \\
1.7071\end{array}$ & $\begin{array}{l}1.8016 \\
1.7980 \\
1.8126\end{array}$ & $\begin{array}{l}2.0239 \\
2.0215 \\
2.0349\end{array}$ & $\begin{array}{l}2.2652 \\
2.2593 \\
2.2703\end{array}$ & $\begin{array}{l}2.5264 \\
2.5134 \\
2.5203\end{array}$ & $\begin{array}{l}2.6649 \\
2.6475 \\
2.6544\end{array}$ & $\begin{array}{l}2.8087 \\
2.7835 \\
2.7934\end{array}$ \\
\hline 70.833 & $\begin{array}{l}1.2734 \\
1.2643 \\
1.2752\end{array}$ & $\begin{array}{l}1.3549 \\
1.3470 \\
1.3584\end{array}$ & $\begin{array}{l}1.4401 \\
1.4339 \\
1.4453\end{array}$ & $\begin{array}{l}1.5291 \\
1.5241 \\
1.5358\end{array}$ & $\begin{array}{l}1.7188 \\
1.7154 \\
1.7292\end{array}$ & $\begin{array}{l}1.9249 \\
1.9208 \\
1.9360\end{array}$ & $\begin{array}{l}2.1482 \\
2.1407 \\
2.1534\end{array}$ & $\begin{array}{l}2.2666 \\
2.2559 \\
2.2678\end{array}$ & $\begin{array}{l}2.3896 \\
2.3738 \\
2.3852\end{array}$ \\
\hline 72.87 & $\begin{array}{l}1.2133 \\
1.2054 \\
1.2131\end{array}$ & $\begin{array}{l}1.2911 \\
1.2844 \\
1.2927\end{array}$ & $\begin{array}{l}1.3724 \\
1.3675 \\
1.3762\end{array}$ & $\begin{array}{l}1.4573 \\
1.4538 \\
1.4616\end{array}$ & $\begin{array}{l}1.6384 \\
1.6368 \\
1.6474\end{array}$ & $\begin{array}{l}1.8352 \\
1.8334 \\
1.8437\end{array}$ & $\begin{array}{l}2.0485 \\
2.0442 \\
2.0564\end{array}$ & $\begin{array}{l}2.1616 \\
2.1545 \\
2.1654\end{array}$ & $\begin{array}{l}2.2791 \\
2.2423 \\
2.2753\end{array}$ \\
\hline 81 & $\begin{array}{l}1.0130 \\
1.0016 \\
1.0062\end{array}$ & $\begin{array}{l}1.0784 \\
1.0692 \\
1.0744\end{array}$ & $\begin{array}{l}1.1467 \\
1.1396 \\
1.1445\end{array}$ & $\begin{array}{l}1.2181 \\
1.2124 \\
1.2183\end{array}$ & $\begin{array}{l}1.3705 \\
1.3668 \\
1.3736\end{array}$ & $\begin{array}{l}1.5361 \\
1.5330 \\
1.5397\end{array}$ & $\begin{array}{l}1.7157 \\
1.7112 \\
1.7234\end{array}$ & $\begin{array}{l}1.8110 \\
1.8048 \\
1.8162\end{array}$ & $\begin{array}{l}1.9101 \\
1.9017 \\
1.9141\end{array}$ \\
\hline 84.3 & $\begin{array}{l}0.9463 \\
0.9331 \\
0.9391\end{array}$ & $\begin{array}{l}1.0075 \\
0.9965 \\
1.0010\end{array}$ & $\begin{array}{l}1.0715 \\
1.0631 \\
1.0666\end{array}$ & $\begin{array}{l}1.1384 \\
1.1322 \\
1.1362\end{array}$ & $\begin{array}{l}1.2811 \\
1.2770 \\
1.2837\end{array}$ & $\begin{array}{l}1.4363 \\
1.4324 \\
1.4399\end{array}$ & $\begin{array}{l}1.6047 \\
1.6002 \\
1.6087\end{array}$ & $\begin{array}{l}1.6940 \\
1.6880 \\
1.6998\end{array}$ & $\begin{array}{l}1.7869 \\
1.7791 \\
1.7897\end{array}$ \\
\hline
\end{tabular}

First line: Formulae values, second line: Chantler values [21], third line: Cullen values [22]. 
Anomalous scattering factors of some rare earth elements

Table 3. Relativistic corrections (high energy limit, $f^{\prime}(\infty)=\Delta$ ).

\begin{tabular}{lccc}
\hline Element & $\Delta_{\mathrm{KP}}$ & $\Delta_{\mathrm{CM}}$ & $\Delta_{\mathrm{CL}}$ \\
\hline Lanthanum & $S$-matrix correction & Multiple correction & Dipole correction \\
Cerium & -0.415 & -0.456 & -0.753 \\
Praseodymium & -0.432 & -0.474 & -0.786 \\
Neodymium & -0.450 & -0.492 & -0.819 \\
Samarium & -0.468 & -0.516 & -0.854 \\
Gadolinium & -0.506 & -0.558 & -0.925 \\
Dysprosium & -0.545 & -0.610 & -1.000 \\
Holmium & -0.586 & -0.648 & -1.079 \\
Erbium & -0.607 & -0.672 & -1.119 \\
\hline
\end{tabular}

$\Delta_{\mathrm{KP}}$ (S-matrix correction): Kissel and Pratt (KP) [20]; $\Delta_{\mathrm{CM}}$ (multipole correction): Creagh and McAuley [18]; $\Delta_{\mathrm{CL}}$ (dipole correction): Cromer and Liberman [15].

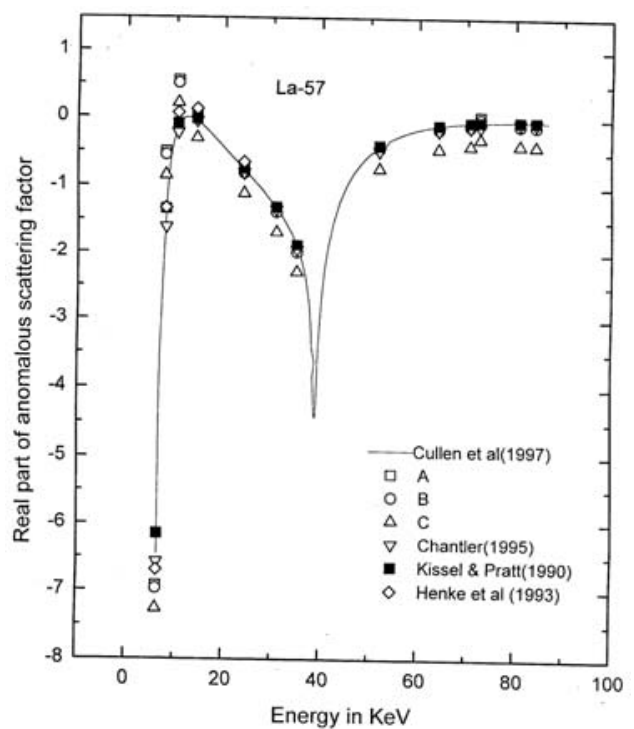

Figure 1. Plot of real part of anomalous scattering factor vs. energy for lanthanum. A: Present values with KP correction, B: present values with CM correction and C: present values with CL correction.

Using the coefficients $a_{i}$ and $b_{i}$, the dispersion integral (6) was calculated analytically for each interval using (7). The integrals $I_{s-1, s}$ and $I_{s, s+1}$ which are not defined are replaced at once by the integral $I_{s-1, s+1}$. Here, $E_{s-1}$ and $E_{s+1}$ are energies very close to the energy of interest $E_{s}$. The final value of the dispersion integral in (3) is obtained simply by adding all $I_{I, I+1}$ values calculated using (7). From these, the $f^{\prime}(E)$ values were calculated according to (3) for each element of interest by separately adding $f^{\prime}(\infty)$ values of Cromer and Liberman (CL) [15], Creagh and McAuley (CM) [18] and Kissel and Pratt (KP) [20]. The values of $f^{\prime}(\infty)$ for each element are shown in table 3. The $f^{\prime}$ values obtained in the case of 


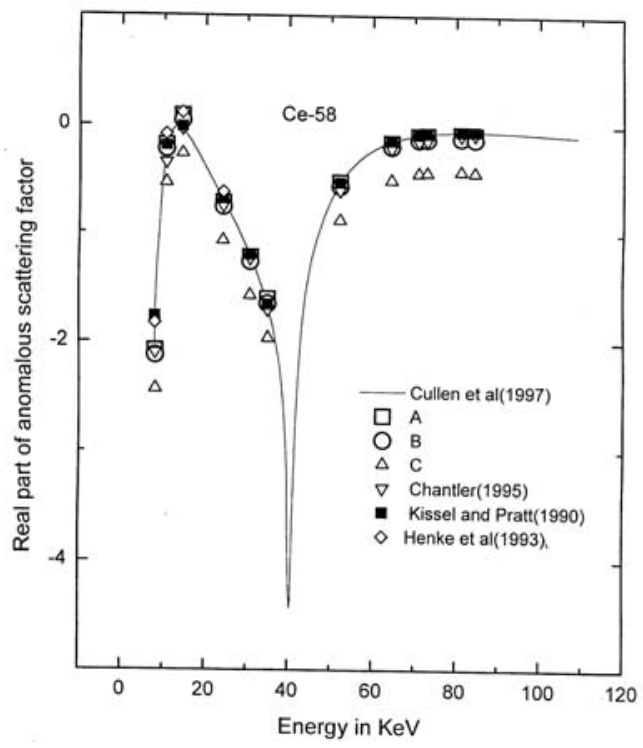

Figure 2. Plot of real part of anomalous scattering factor vs. energy for cerium. A: Present values with KP correction, B: present values with $\mathrm{CM}$ correction and C: present values with CL correction.

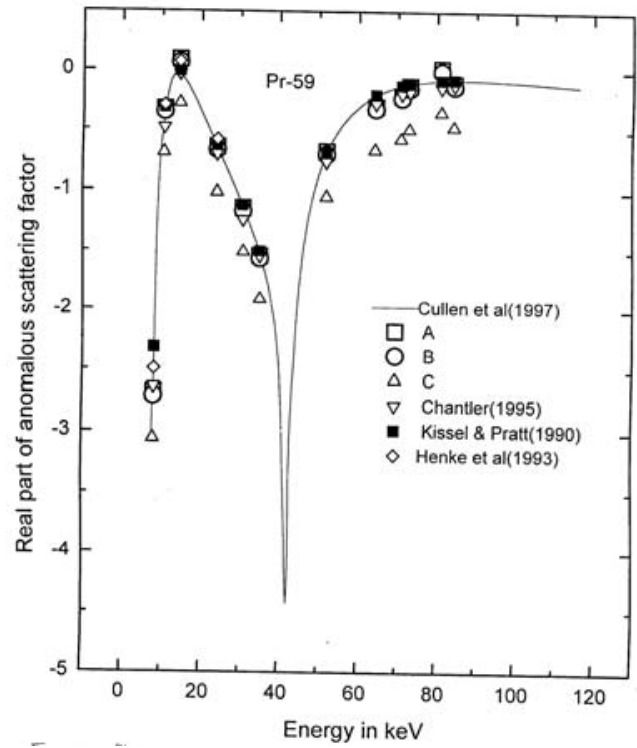

Figure 3. Plot of real part of anomalous scattering factor vs. energy for praseodymium. A: Present values with KP correction, B: present values with CM correction and C: present values with CL correction. 
Anomalous scattering factors of some rare earth elements

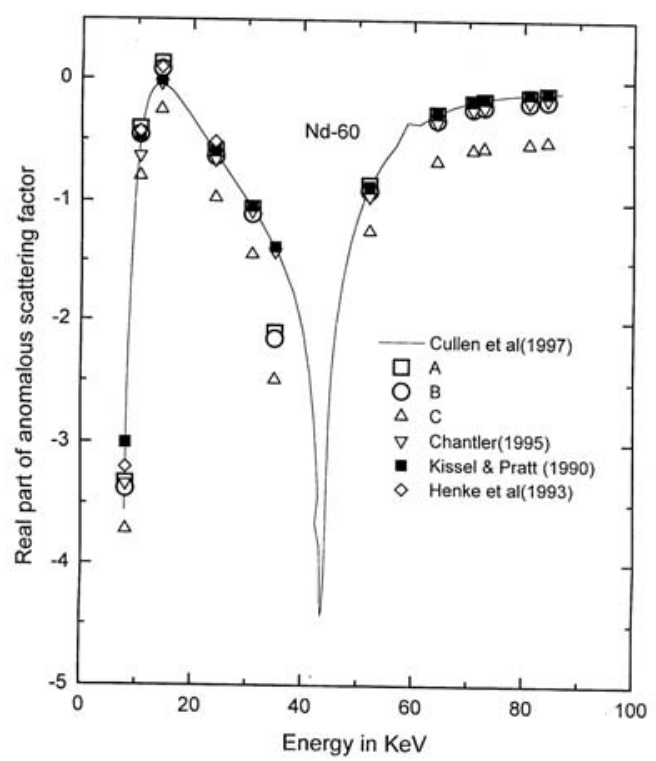

Figure 4. Plot of real part of anomalous scattering factor vs. energy for neodymium. A: Present values with KP correction, B: present values with $\mathrm{CM}$ correction and C: present values with CL correction.

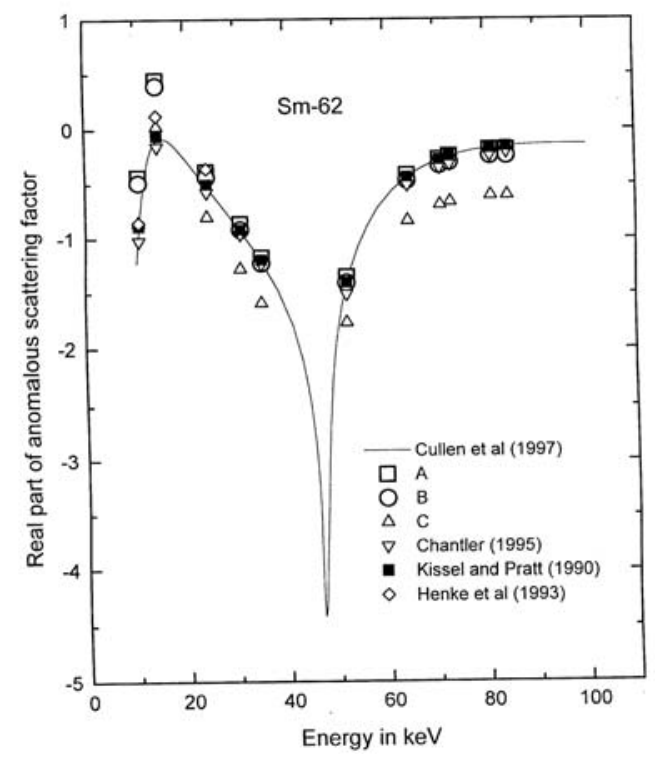

Figure 5. Plot of real part of anomalous scattering factor vs. energy for samarium. A: Present values with KP correction, B: present values with CM correction and C: present values with CL correction. 
S B Appaji Gowda et al

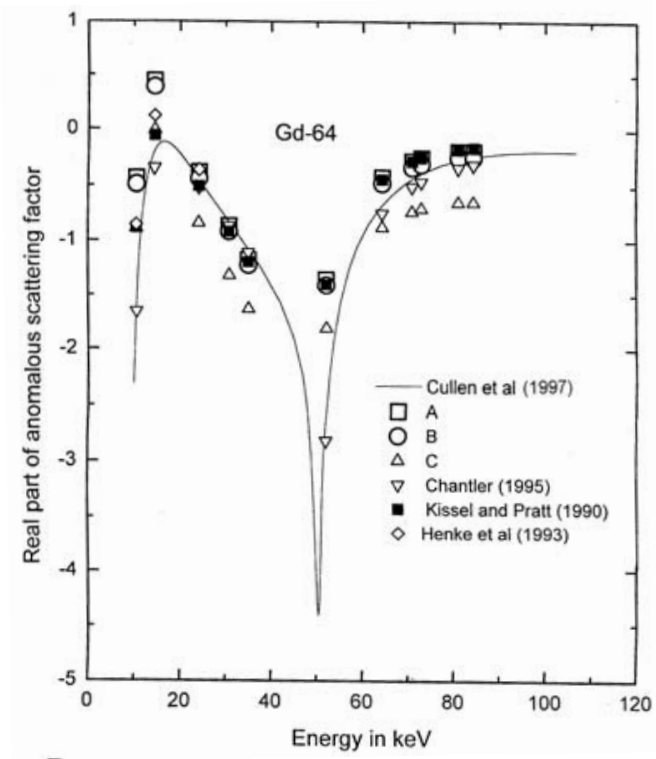

Figure 6. Plot of real part of anomalous scattering factor vs. energy for gadolinium. A: Present values with KP correction, B: present values with $\mathrm{CM}$ correction and C: present values with CL correction.

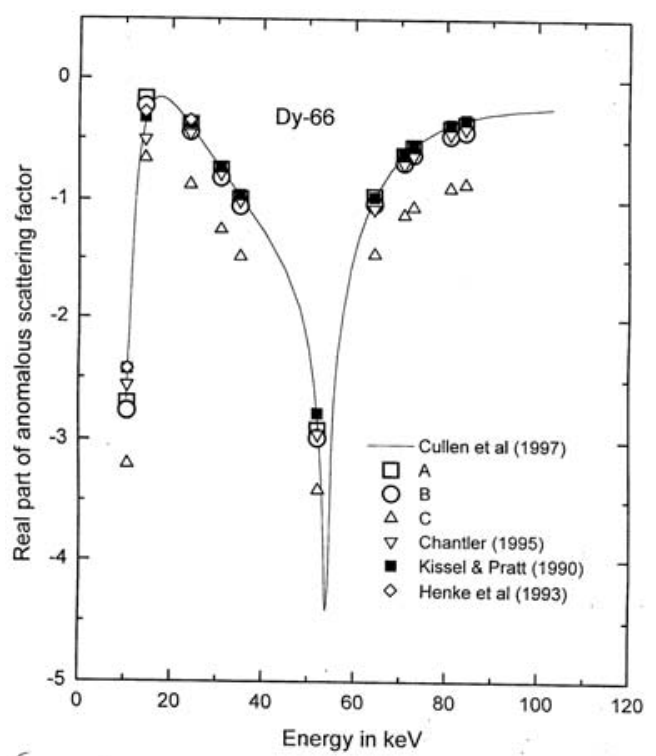

Figure 7. Plot of real part of anomalous scattering factor vs. energy for dysprosium. A: Present values with KP correction, B: present values with $\mathrm{CM}$ correction and C: present values with CL correction. 
Anomalous scattering factors of some rare earth elements

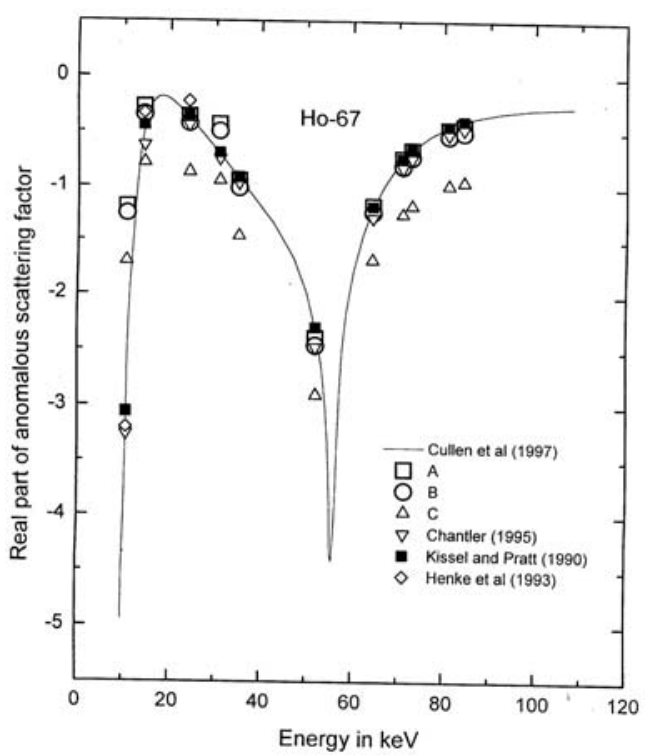

Figure 8. Plot of real part of anomalous scattering factor vs. energy for holmium. A: Present values with KP correction, B: present values with CM correction and C: present values with CL correction.

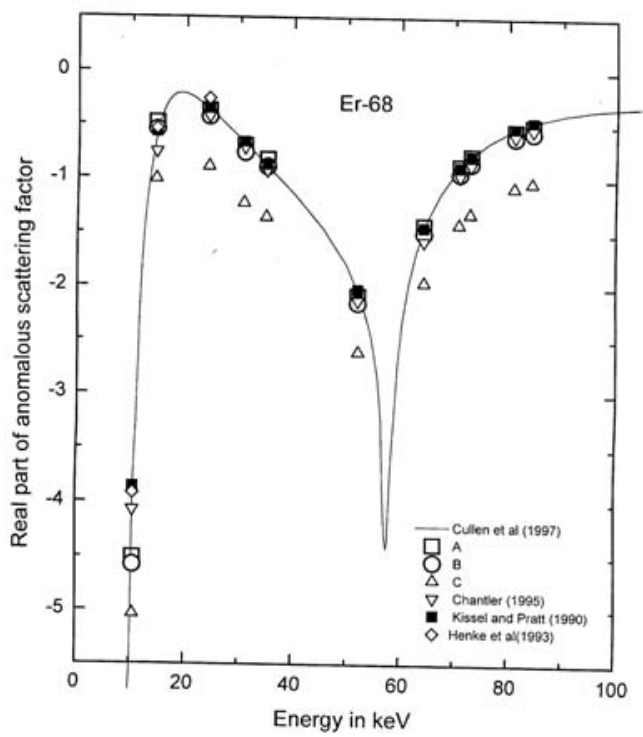

Figure 9. Plot of real part of anomalous scattering factor vs. energy for erbium. A: Present values with KP correction, B: present values with CM correction and C: present values with CL correction. 


\section{S B Appaji Gowda et al}

the elements $\mathrm{La}, \mathrm{Ce}, \mathrm{Pr}, \mathrm{Nd}, \mathrm{Sm}, \mathrm{Gd}$, Dy, Ho and Er are shown in figures 1 to 9 along with the theoretical data of Cullen et al [22] (KP), Chantler [21] (CM) and the compiled data of Henke et al [6]. It can be seen from the figures that the present values of $f^{\prime}$ in which CM or KP correction is included follow the trend suggested by theory.

\section{Conclusions}

Based on this study, reasonable values of the dispersion corrections $f^{\prime}(E)$ and $f^{\prime \prime}(E)$ could be obtained from a fairly accurate total attenuation cross-section data set measured in a narrow beam good geometry set-up by employing a high resolution detector to detect photons emitted from radioisotopes. The present study points to the fact that within the range of experimental errors, the real part of the dispersion corrections to which the relativistic corrections calculated by Kissel and Pratt ( $S$-matrix approach) or Creagh and McAuley have been included are in better agreement with the theoretical values. It is felt that the simple formulae for $f^{\prime \prime}$ values in terms of $Z$ and $E$ help in the speedier calculation of $f^{\prime \prime}$ below, above and at the $K$-edge of any element in the range $Z=57-68$ and $E=10-100$ $\mathrm{keV}$ to a good accuracy.

\section{References}

[1] H M Nussenzveig, Causality and dispersion relations (Academic Press, New York, 1972) p. 183

[2] L Kissel, Acta Crystallogr. A51, 271 (1995)

[3] M S Wang, Phys. Rev. A34, 636 (1986)

[4] M S Wang and R H Pratt, Phys. Rev. A28, 3115 (1983)

[5] B Zhou, Lynn Kissel and R H Pratt, Nucl. Instrum. Methods Phys. Res. B66, 307 (1992)

[6] B L Henke, P Lee, T J Tanaka, R L Shimambukuro and B K Fujikawa, At. Data Nucl. Data Tables 27, 1 (1982)

B L Henke, E M Gullikson and J C Davis, At. Data Nucl. Data Tables 54, 181 (1993)

[7] I Waller, Z. Phys. 51, 213 (1928)

[8] M Gavrila, Photon atom elastic scattering in inner-shell and $x$-ray physics of atoms and solids edited by B Craseman (Plenum, New York, 1981)

[9] P P Kane, L Kissel, R H Pratt and S C Roy, Phys. Rep. 140, 75 (1986)

[10] D Creagh, J. Phys. 38, 371 (1985)

[11] H Hönl, Ann. Phys. 18, 625 (1933)

[12] R W James, The optical principles of the diffraction of $x$-rays (Bell and Sons, London, 1962) ch. 4

[13] Ramaseshan and S C Abrahams, Proceedings of the International Conference, 22-26 April 1974 (Madrid, Spain, 1975)

[14] D Templeton, in Resonant anomalous $x$-ray scattering edited by Matelik et al (Elsevier, NorthHolland, 1994) p. 9

[15] T Cromer and D Liberman, J. Chem. Phys. 53, 1891 (1970)

[16] D Creagh, Phys. Lett. A103, 52 (1984)

[17] D Y Smith, Phys. Rev. 35, 3381 (1987)

[18] D C Creagh and W J McAuley, International tables for crystallography C edited by A J C Wilson (Kluwer Academic Publishers, Dordrecht, 1992) p. 206

[19] L Kissel, S C Roy and R H Pratt, Phys. Rev. A22, 1972 (1980) 
[20] L Kissel and R H Pratt, Acta Crystallogr. A46, 170 (1990)

[21] C T Chantler, J. Phys. Chem. Ref. Data 24, 71 (1995)

[22] D E Cullen, E Dermott, John H Hubbell and L Kissel, The Evaluated Photon Data Library, 97 Version (Lawrence Livermore National Laboratory, 1997)

[23] T Fukamachi, S Hosoya, T Kawamura and J Hastings, J. Appl. Crystallogr. 10, 321 (1977)

[24] U Bonse, I Hartmann-Lotsch and H Lotsch, Nucl. Instrum. Methods 208, 603 (1983)

[25] D Creagh, Phys. Lett. A77, 129 (1980)

[26] P Drier, P Rabe, W Malzfeldt and W Niemann, J. Phys. C17, 3123 (1984)

[27] L Gerward, G Thuesen, Stibius Jensen and I Alistrup, Acta Crystallogr. A35, 852 (1979)

[28] M Keffi, J M Andre, Y Heno, G Gorgi and C Bonnelle, Phys. Rev. 45, 2859 (1992)

[29] J H Barkyoumb and D Y Smith, Phys. Rev. 41, 4863 (1990)

[30] Sandiago, T K Umesh and Ramakrishna Gowda, Pramana - J. Phys. 48, 1077 (1997)

[31] T K Umesh, Sandiago and Ramakrishna Gowda, Radiat. Phys. Chem. 51, 373 (1998)

[32] M J Berger and J H Hubbell, Natl. Bur. Stand. Internal Rep. 87, 3597 (1987); XCOM, photon cross section on a personal computer

[33] M L Mallikarjuna, S B Appajigowda, R Gowda and T K Umesh, Radiat. Phys. Chem. 65/3, 217 (2002)

[34] T K Umesh, R Gowda and B Sanjeevaiah, Phys. Rev. A25, 1986 (1982)

[35] T K Umesh, C Ranganathaiah and B Sanjeevaiah, Phys. Rev. A29, 387 (1984)

[36] T K Umesh, C Ranganathaiah and B Sanjeevaiah Phys. Rev. A32, 959 (1985)

[37] T K Umesh, S J Anasuya, J Shylajakumari, Channegowda, Gopinathan Nair and Ramakrishna Gowda, Phys. Rev. A45, 2101 (1992)

[38] J H Scofield, Theoretical photoionization cross-sections from 1-1500 keV (Lawrence Livermore Laboratory, 1973) UCRL-51326 